International Journal of Wireless \& Mobile Networks (IJWMN) Vol. 6, No. 1, February 2014

\title{
IMPACT OF DIFFERENT MOBILITY SCENARIOS ON FQM FRAMEWORK FOR SUPPORTING MULTIMEDIA APPLICATIONS IN MANETS
}

\author{
Mohammed Saghir \\ Hodeidah University, Yemen
}

\begin{abstract}
In Mobile Ad hoc Network (MANET), the mobility of nodes is a challenging issue for designers. There are lots of possibilities of mobile scenarios in this kind of network. The source, destinations and intermediate nodes may not be using the same mobile scenarios. In this study, three mobile scenarios are taken in consideration and these scenarios are source mobility, destinations mobility and intermediate nodes mobility. The impact of the three mobile scenarios on the Quality of service Multicast Framework (FQM) for supporting multimedia applications in MANETs is studied. The simulation results show that mobility of group of destinations affects the performance of FQM framework more than mobility of source. In addition, the analysis of simulation results shows that mobility of intermediate nodes does not have high effect on the performance of FQM framework when node density is not high.
\end{abstract}

\section{KEYWORDS}

MANET, Mobility, Scenarios, density\& FQM.

\section{INTRODUCTION}

The environment for Mobile Ad hoc Networks (MANETs) is very volatile so connections can be dropped at any moment. Distant nodes communicate over multiple hops and therefore nodes must cooperate with each other to provide routing. Among types of wireless networks, MANET provides flexible communication with low cost. All communications are done over wireless media without the help of wired base stations. The challenges in MANETs are attributed to mobility of relay nodes, absence of routing infrastructure, low bandwidth and computational capacity of mobile nodes. The mobility of nodes affects the link sate and varies the number of nodes entering or leaving the neighborhood [1].

The applications of ad hoc networks are finding in several areas due to its quick and economic deployment. These applications are including military applications, emergency operations, meeting applications, law enforcement applications, collaborative and distributed applications. For military applications, mobile ad hoc networks can provide the required communication between groups of soldiers in unknown area where install fixed infrastructure may be impossible. In the emergency operations such as search and rescue, mobile ad hoc networks are very useful for establishing communication where the conventional infrastructure communications are destroyed duo to a war or earthquake. Mobile ad hoc network also useful for meeting applications where students in the class, researchers in conference or business people need to establish a meeting through voice conversation, video chatting or video conferencing. Ad hoc networks can be used to support collaborative and distributed applications where the decision of one participant depends on the current environmental conditions and on the actions of other users. An example of this type of applications is the coordination between employers in rescue agency, where the operations are based on the conditions of all affected areas. The shared characteristics of these applications are team collaboration of large number of mobile nodes, limited bandwidth, the need 
for supporting multimedia applications and low latency access to distributed resources as distributed database access for situation awareness in the battlefield [2]. These applications are performed in one-to-many or many-to-many communications so multicasting is very important technique for these applications.

The mobile ad hoc network is expected to be deployed in different types of environments. These environments include cities, universities, highways, markets, conferences and battlefields. The most common in these environments is the presence of obstacles that block node movement and that prevent propagation of wireless signals. Examples of obstacles include buildings, mountains, hillsides and cars.

The mobile nodes in many real life applications move in groups while others move individually and independently [3]; mobility correlation among nodes is quite common. Moreover, node mobility in real military scenarios is not always independent. In the battlefield, nodes with the same mission usually move in groups such as tank battalions or swarms in Unmanned Aerial Vehicles (UAV) networks [4].

Mobile nodes can be classified into multiple classes as walker and cars. Each class of mobile nodes has different requirements such as moving speed. In such cases, different groups of mobile nodes can be defined and only mobile nodes that belonging to the same moving speed class can merge into a group. The communications in ad hoc networks are often among teams which tend to coordinate their movements such as firemen rescue team, flood rescue team, earthquake rescue team in a disaster recovery situation and search and rescue team in law enforcement. For this, the need arises for developing efficient and realistic group mobility models. From all these requirements of classes, it is clear that mobility models are application dependent. Moreover, the various mobility models are expected to affect the performance of different MANET network protocols in different ways. Multicast protocols are being tested as they stand to get the most impact from group mobility [2].

The motivation for supporting QoS multicasting in mobile ad hoc networks is the fact that multimedia applications are becoming important for group communication. Most of the multicast applications can potentially involve in different scenarios with different mobility model dependent on the environment and the nature of the interactions among the participants in the multicast group [5].

Real-time applications over wireless ad hoc networks include video conferencing at a location without wireless infrastructure, transmitting video on the battlefield, and search and rescue operations after a disaster. Real-time applications are fundamentally different from best-effort applications, since interactive real-time applications are delay and loss packet sensitive. The later real-time packets will be dropped while best-effort packets can be accepted. Therefore, the retransmission techniques are not generally applicable to real-time interactive applications, especially in multicast situations [6]

This paper is structured as follows: Section 2 gives an overview on the previous work whereas Section 3 gives an overview on the QoS multicast framework FQM and defines the three mobile scenarios. In Section 4, the simulation results of implementing FQM with the three mobile scenarios and different node density are presented. Finally, Section 5 provides the conclusions of this study and gives some suggestions for future work. 
International Journal of Wireless \& Mobile Networks (IJWMN) Vol. 6, No. 1, February 2014

\section{Literature RevieW}

A mobility model represents nodes distribution and movement over the network. Different studies have approved that a selection of mobile scenario can affect the performance of routing protocols in MANETs. The presence of obstacles in many mobile ad hoc network environment blocks node movement and prevents propagation of wireless signals. The people in that environment mostly travel frequently between buildings located physically close to each other while people travel less frequently to buildings further away. The traffic in that environment is concentrated in a specific area more than others. The university center is likely to be a popular destination where general services are available. Furthermore, restaurants, concerts, lectures and special events in university can all act as attraction points where students from all areas of university flow to one area at a specified time. The mobility model for that scenario is investigated and the impact on throughput and network performance resulting from such concentrated traffic areas is studied [7]. The simulation results of the obstacle model and the random waypoint model show that the two mobility models significantly impact the performance of an ad hoc network routing protocols. In addition, the results have shown that the mobility model affects a variety of characteristics, including the connectivity of the nodes and network density, as well as the packet delivery ratio and control overhead of the routing protocol.

In [8], the performance degradation due to rapidly time varying channels in a repetition based coherent cooperative system is investigated and two cooperative scenarios with static forward nodes are studied. In first scenario source node is mobile while in second scenario destination node is mobile. A detection rules is developed for a variety of mobile scenarios. The detection rules that take into account the mobility of the nodes are mostly hybrids of partially coherent detectors and non-coherent detectors. The results of implementing the two scenarios with detection rules show that source mobility affects the performance slightly more than destination mobility for both amplify and forward (AF) and demodulate and forward (DF) relays, despite the symmetry of the network.

The virtual track based group mobility model (VT) which closely approximates the mobility models in military MANET scenarios is proposed [4]. Different types of node mobility are defined when nodes are moving in group, nodes are moving individually and nodes are static. In large scale military scenarios, mobility coherence among nodes is quite common. Moreover, the VT model also models the dynamics of group mobility when mobile nodes can merge or split. The results show that performance of routing protocols under the group mobility model can be enhanced by individual nodes and static nodes. When individual nodes are randomly distributed, the connectivity among multiple groups is increased. The network performance is improved significantly in a military scenario with dominant group mobility and deploying forwarding nodes. Furthermore, the performance of mobile ad hoc network is based on the type of mobility model.

The Reference Point Group Mobility (RPGM) is introduced to represent the relationship among mobile nodes [2]. RPGM can be applied to many existing applications. Moreover, by proper choice of parameters, RPGM can be used to model several previously proposed mobility models. This study investigates the impact of the mobility model on the performance of a specific network protocols. The RPGM model applied to two different network protocol scenarios, clustering and routing. The network performance has evaluated under different mobility patterns and for different protocol implementations. The results indicate that different mobility model affect the various protocols in different ways. Furthermore, the quality of routing protocol is based on choice of mobility model. 
International Journal of Wireless \& Mobile Networks (IJWMN) Vol. 6, No. 1, February 2014

The impact of human mobility on the link and route lifetime in mobile ad hoc network is studied and analyzed in [9]. In addition, the differences between the effect of mobility model and collisions/interference are studied. Many experiments are conducted in a typical office environment. In these experiments, twenty Personal Digital Assistants (PDAs) are distributed with IEEE $802.11 \mathrm{~b}$ wireless interfaces to group of students and researchers to represent different test users. The users working on the same floor in a building and as result users will be in wireless transmission range most of the time. The results show that interruptions due to human mobility and collisions/interference have a completely different impact on the lifetime of links and routes.

The Bypass-AODV is a new optimization of the AODV routing protocol for mobile ad hoc networks. It is proposed a local recovery mechanism to enhance the performance of the AODV routing protocol. The Bypass-AODV shows better performance than AODV with random waypoint mobility model. However, random waypoint is a simple model that may be applicable to some scenarios but it is not sufficient to capture some important mobility characteristics of other scenarios. The performance of Bypass-AODV under a different mobility models including group mobility models and vehicular mobility models is investigated in [10]. The results of simulation show a comparable performance for Bypass-AODV and AODV protocols for group mobility model while for vehicular mobility models; Bypass-AODV suffers from performance degradation in high-speed conditions.

In [11], three different kinds of node mobility situations are taken in considerations which are source node mobility, destination node mobility and whole network nodes mobility. For all these mobility situations, both reactive mode of packet transformation and proactive mode of packet transformation are taken for comparison. The three mobility situations are studied with two different Protocols namely DSR [12] and DSDV [13]. The conclusion of the work is that when source is needed to move completely, proactive mode routing (DSDV) is used. The reactive mode is preferred for the network where complete mobile network is needed. In addition, when overall delay is considered then the Proactive mode routing (DSDV) is selected.

The performance of AODV routing protocol is studied under three different mobility models [14]. In addition, a new measurement technique called probability of route connectivity is introduced. This technique is used to quantify the success rate of route established by a routing protocol. The performance of AODV routing protocol is evaluated under several link conditions. Results clearly show that mobility models affect the performance of AODV routing protocols.

The impact of mobility models on the performance of multicast routing protocols in MANET is studied and analyzed [5]. The Random Way Point, Reference Point Group and Manhattan mobility models are used as mobility models and the On-Demand Multicast Routing Protocol (ODMRP) [15][16], Multicast Ad hoc On-demand Distance Vector Routing protocol (MAODV)[17][18] and Adaptive Demand driven Multicast Routing protocol (ADMR) [19] are used as multicast routing protocols. The results of implementing three widely used mobility models and the three multicast routing protocols in NS2 have shown that different mobility models have different affect on the performance of the multicast routing protocols.

In [20], the impact of mobility predictive models on the parameters of mobile nodes such as the arrival rate and the size of mobile nodes using Pareto and Poisson distributions is investigated. The results show that when the arrival rate increases, the mobile nodes population also increases. The Pareto distribution was considered because the Poisson distribution is not accurate for the arrival distribution. In addition, the results show that the two-parameter Pareto distribution performed better than the single-parameter Pareto distribution and exponential distribution.

Previous studies have focused on the impact of mobility models on the performance of routing protocols where all network mobile nodes use the same mobility scenario. Although some previous studies [8][11] have implemented different mobility scenarios for unicast routing 
International Journal of Wireless \& Mobile Networks (IJWMN) Vol. 6, No. 1, February 2014

protocol in mobile ad hoc networks, this study focuses on the impact of different mobility scenarios for source nodes, intermediate nodes and destination nodes for multicast routing protocol in mobile ad hoc networks.

\section{THE FQM WITH THE THREE CLASSES OF MOBILE SCENARIOS}

In this section, an overview on the QoS Multicast Framework (FQM) is given and three classes of mobile scenarios are described.

\subsection{The FQM QoS multicast framework}

Multicast routing is more efficient in MANETs because it is inherently ready for multicast due to their broadcast nature that avoids duplicate transmission. Packets are only multiplexed when it is necessary to reach two or more destinations on disjoint paths. This advantage conserves bandwidth and network resources [21]. A cross-layer framework FQM is proposed to support QoS multicast applications for MANETs [22]. The FQM framework consists of five components. The first component of the framework is a new and efficient QoS multicast routing protocol (QMR) which is used to find and maintain the paths that meet the QoS requirements. The second component is a distributed admission control which used to prevent nodes from being overloaded by rejecting the request for new flows that will affect the ongoing flows. The third component is an efficient way to estimate the available bandwidth and provides the information of the available bandwidth for other QoS schemes. The fourth component is a source based admission control witch used to prevent new sources from a affecting the ongoing sources if there is not enough available bandwidth for sending to the all members in the multicast group. The fifth component is a cross-layer design with many QoS scheme: classifier, shaper, dynamic rate control and priority queue.

The traffic is classified and processed based on its priority; therefore, control packets and realtime packets will bypass the shaper and sent directly to the interface queue at MAC layer. The best-effort packets should be regulated based on the dynamic rate control. In the priority scheduling, control packet, data packets and best-effort are maintained in separate queues in FIFO order. In the scheduling algorithm, the packet with high priority should be sent firstly to the channel. Control packets should have highest priority, while real-time packets should have higher priority than best-effort packets. These schemes work together to support real-time applications. The various mobile scenarios are expected to affect the performance of different network protocols in different ways, furthermore, the mobility models are application dependent. Three mobile scenarios are described in the following sections.

\subsection{Scenario one (source mobility):}

The source node in this scenario is moving according to random waypoint mobility model while destination nodes and intermediate nodes are static. The applications for sensor networks may represent this scenario. In addition, leader of groups for military in battlefield such as swarms or tank battalions also represents this scenario.

\subsection{Scenario two (destinations mobility):}

In this scenario, destinations are moving according to the random waypoint mobility model while source and intermediate nodes are static. Students in distance learning lectures and members in audio or video conferences may represent this scenario. The destinations may move around a static source. In addition, vehicle cars and marketing people also represent this kind of scenario. 
International Journal of Wireless \& Mobile Networks (IJWMN) Vol. 6, No. 1, February 2014

The vehicle cars move around base stations in the road and marketing people may move around base station in the market.

\subsection{Scenario three (intermediate nodes mobility):}

The intermediate nodes in this scenario are moving according to random mobility models while source and destinations are static. In some video conferencing, source and destinations are static in some places and the mobility was represented by intermediate nodes that can forward data traffic without submitting to the video conferencing.

\section{Performance Evaluation}

In this section, the impact of different mobility situations on the FQM framework for supporting multimedia applications in MANETs is studied using GLOMOSIM [23]. Several mobile scenarios are used and the simulation was run using a MANET with fixed number of nodes moving over a rectangular $1000 \mathrm{~m} \times 1000 \mathrm{~m}$ area for over 900 seconds of simulation time. In each mobile scenario, the mobile nodes are moved according to the random waypoint mobility model provided by GLOMOSIM. The Random Waypoint mobility model is flexible and can be used to create a real mobility scenario for the moving of people in many environments [24]. Mobility speed was $20 \mathrm{~m} / \mathrm{s}$ and the pause time was $0 \mathrm{~s}$. The radio transmission range was $250 \mathrm{M}$ and the channel capacity was $2 \mathrm{Mbit} / \mathrm{s}$. Each data point in this simulation represents the average result of ten runs with different initial seeds.

The impact of different mobile scenarios on the performances of FQM for supporting multimedia applications is studied through the following performance metrics:

- Packet delivery ratio: the average of the ratio between the number of data packets received and the number of data packets that should have been received at each destination. This metric indicates the reliability of the proposed framework.

- The Control overhead: the number of transmitted control packet (request, reply, acknowledgment) per data packet delivered. Control packets are counted at each hop. The available bandwidth in MANETs is limited so it is very sensitive to the control overhead.

- Average latency: the average end-to-end delivery delay is computed by subtracting packet generation time at the source node from the packet arrival time at each destination. The multimedia applications are very sensitive to the packet delay; if the packet takes long time to arrive at destinations, it will be useless and will be dropped.

- Jitter: the variation in the latency of received packets. It is determined by calculating the standard deviation of latency. This is an important metric for multimedia applications and should be kept to a minimum value; a smaller value indicates a higher quality flow.

- Group Reliability: the ratio of number of packets received at 95\% of destination and number of packets should be received. This means that the packet is considered to be received only if it is received by $95 \%$ of the number of multicast group.

\subsection{The Performance of FQM under different mobile scenarios}

In this section, the impact of the three mobile scenarios on the performance of the FQM framework for supporting multimedia applications in MANETs under different performance metrics is studied. 


\subsubsection{Packet Delivery Ratio (PDR)}

The PDR as a function of the three mobile scenarios is given in Figure 1. The PDR for scenario 1 and scenario 3 is higher than PDR for scenario 2. This is because the mobility of group of destinations with low node density for scenario 2 affects PDR more than mobility of source in scenario 1 and mobility of intermediate nodes in scenario 3. Although, it has been proven that mobility of source affects PDR more than mobility of destination [8] and this for one source and one destination (unicast routing) whereas in these scenarios (multicast routing) group of destinations affect PDR more than one source. In addition, the PDR for scenario 3 is the highest because only intermediate nodes are mobile. The speed of mobility of intermediate nodes does not affect PDR very high because Forward Nodes (FNs) can be selected from intermediate nodes periodically every 3 seconds.

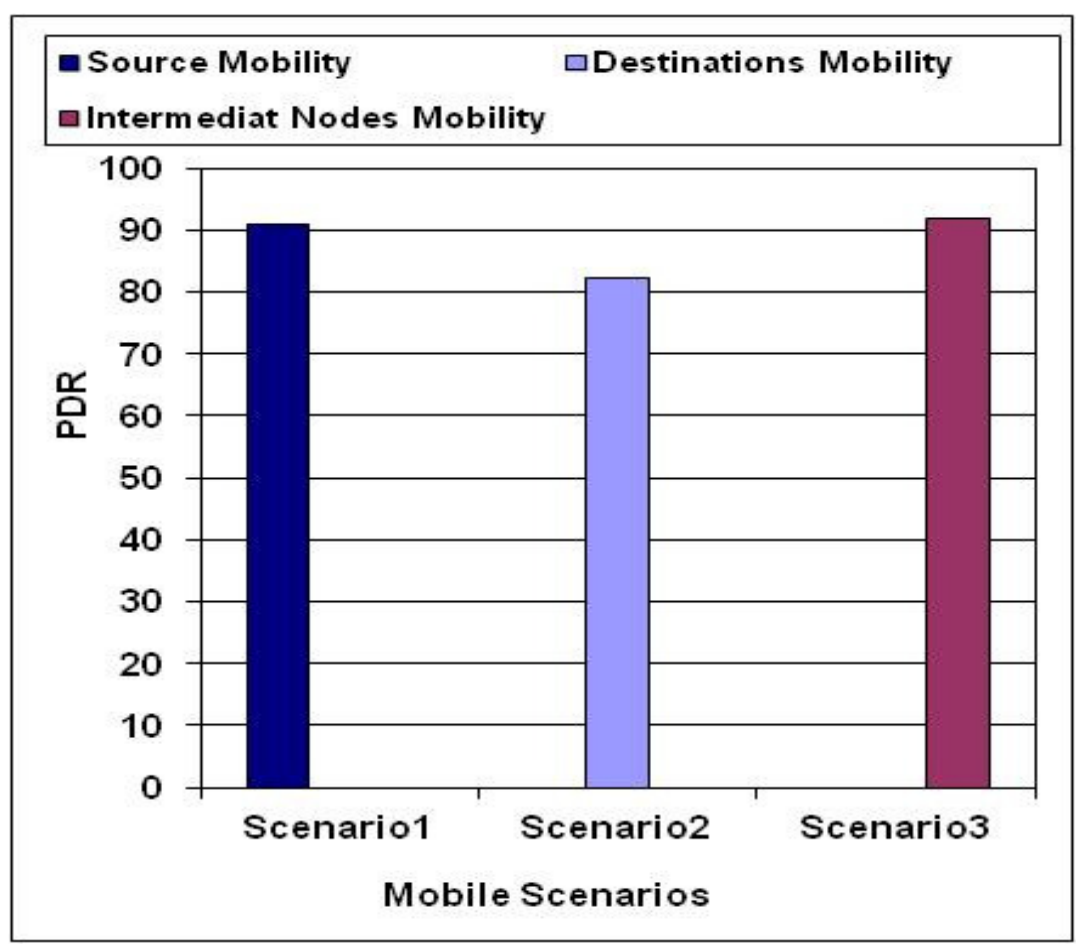

Figure 1. Performance of PDR vs. mobile scenarios 


\subsubsection{Control Overhead $(\mathrm{OH})$}

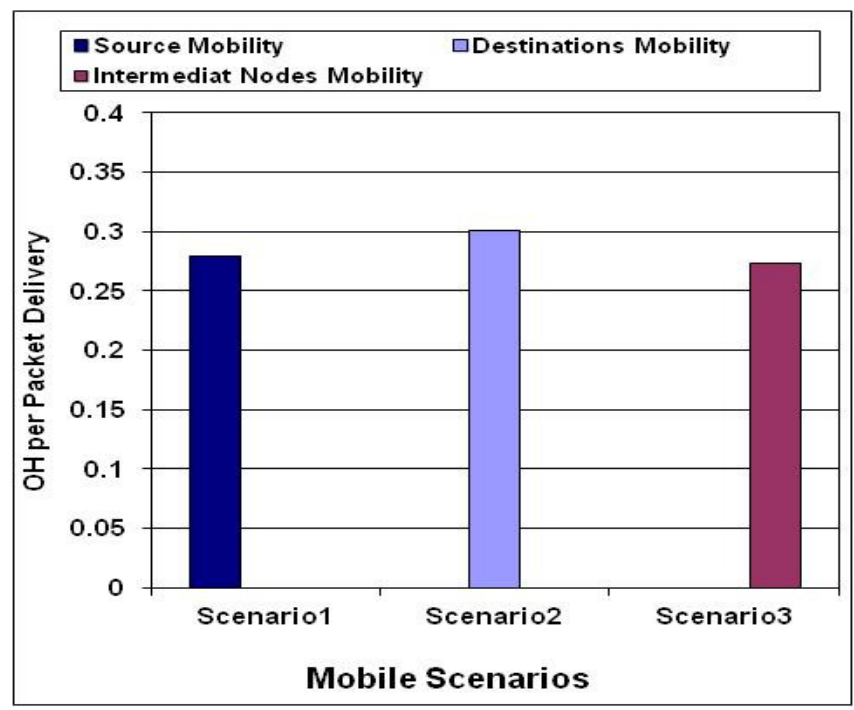

Figure 2. Performance of $\mathrm{OH}$ vs. mobile scenarios

The $\mathrm{OH}$ as a function of the three mobile scenarios is given in Figure 2 which shows that the average control overhead for scenario 2 is higher than average control overhead for scenario 1 and scenario 3. This is because the number of data packets that received at destinations for scenario 1 and scenario 3 is higher than the number of data packets that received at destinations for scenario2 as described in Section 4.1.1. In addition, the mobility in the three scenarios does not affect the control overhead because the source node sends control packets periodically every 3 seconds and as a result the number of generated control packets in the three mobility scenarios almost the same. Furthermore, the differences between the PDR in the three scenarios are reflected in the average of control $\mathrm{OH}$.

\subsubsection{Average Latency (AL)}

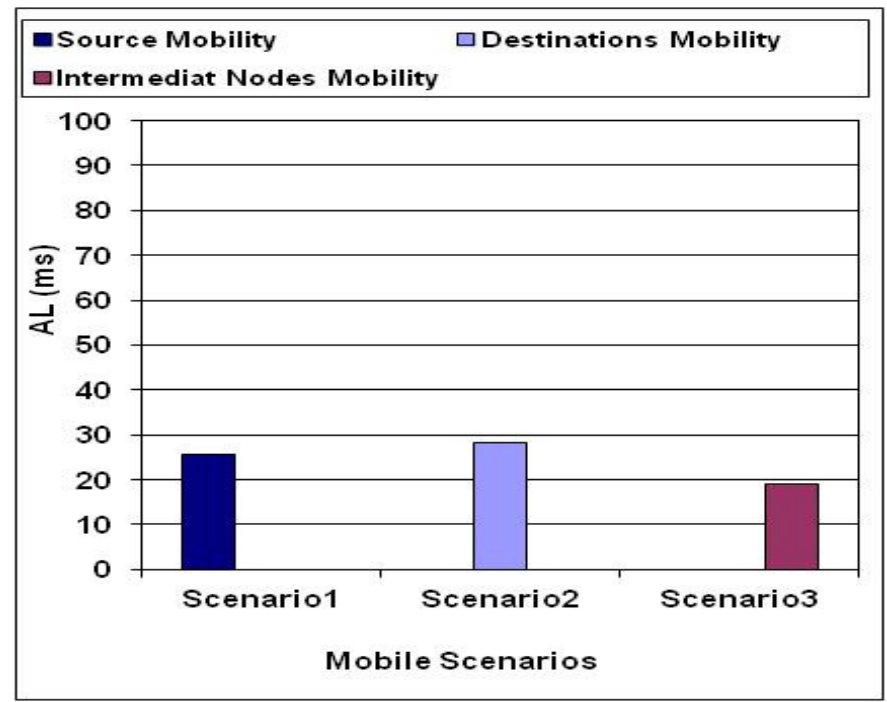

Figure 3. Performance of AL vs. mobile scenarios 
The AL as a function of the three mobile scenarios is given in Figure 3. The AL for scenario 2 is higher than AL for scenario 1 and scenario 3 because the mobility of destinations in scenario 2 affects paths from source to destinations and as a result data packets take long time to arrive at destinations after destinations movements. Although source node and intermediate nodes are static, new FN nodes are needed to be selected and as a result new paths to destinations will be constructed and for this, average latency increased. In addition, the AL for scenario 3 is the lowest because source and destinations are static and as a result latency for destination nodes that received directly from source node does not affected by mobility of intermediate nodes.

\subsubsection{Jitter}

Figure 4 reflects the jitter as a function of the three mobile scenarios. The figure reflects that jitter for scenario 2 is higher than jitter for scenario 1 and scenario 3. This is because mobility of destinations in scenario 2 has a high effect on the constructed paths from source to destinations more than mobility of source in scenario 1 as discussed in Section 4.1.3. In addition, the jitter in scenario 3 is very low because only intermediate nodes are mobile. The mobility of intermediate nodes in scenario 3 does not have high effect on the average latency and as a result it does not have high effect on the jitter. Furthermore, the data packet latency for destination nodes that received from source directly does not change and as a result the jitter is very low.

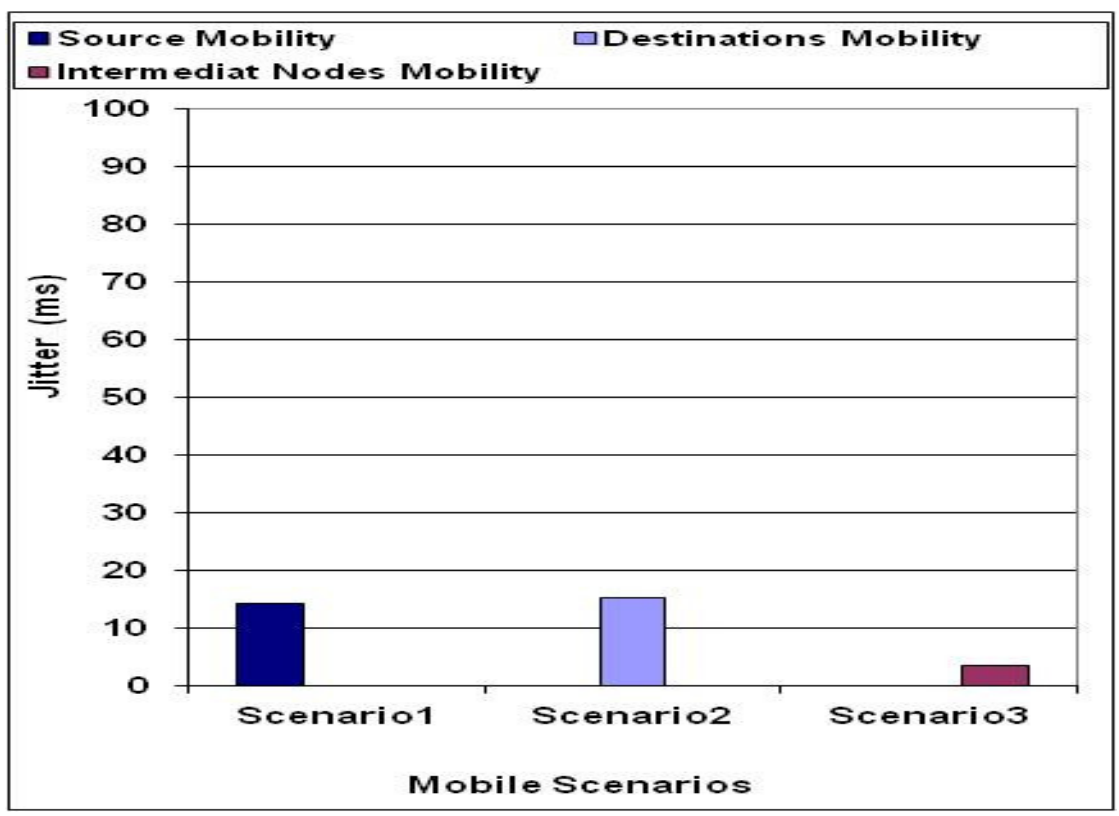

Figure 4. Performance of Jitter vs. mobile scenarios 


\subsubsection{Group Reliability (GR)}

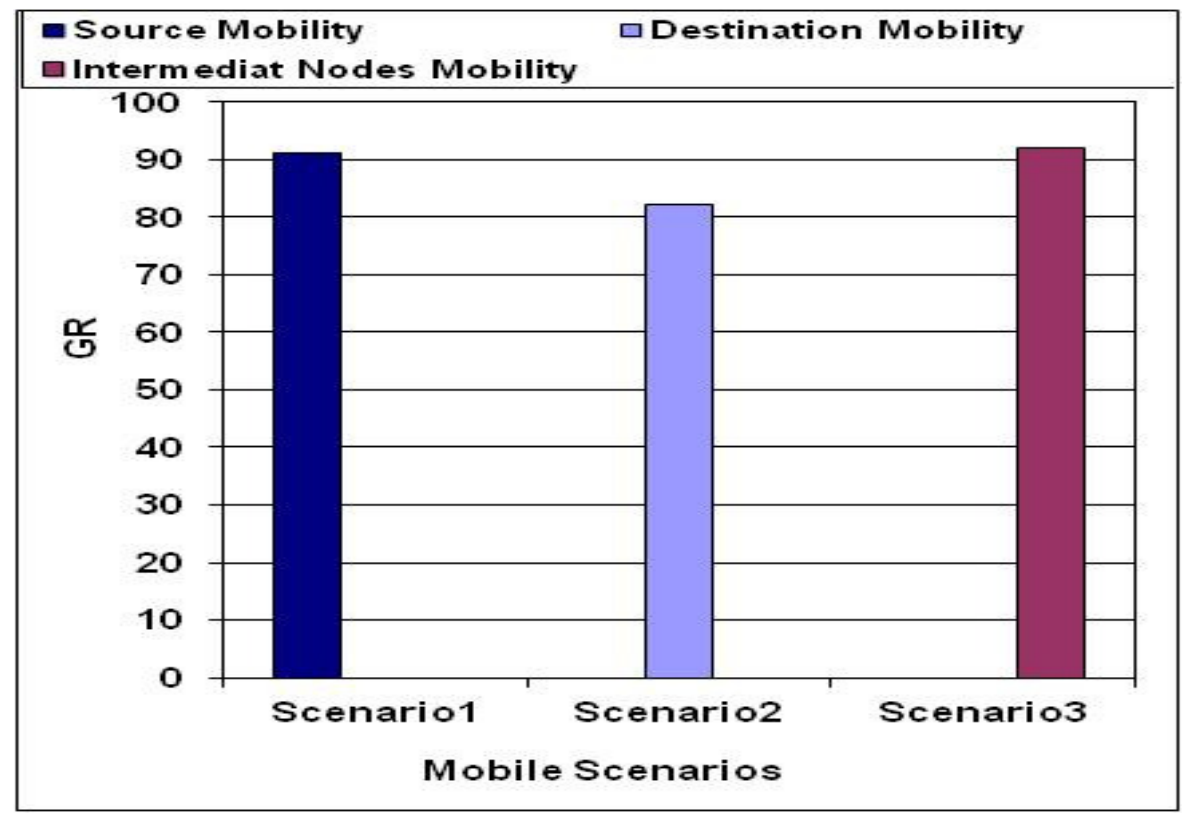

Fig.5: Performance of GR vs. mobile scenarios

The GR as a function of the three mobile scenarios is given in Figure 5. The figure shows that GR for scenario 1 and scenario 3 are higher than GR for scenario 2. As discussed in Section 4.1.1, The mobility of destinations with low node density in scenario 2 affects data packets that received at destinations and as a result some data packets are dropped before they arrive at all destinations in the multicast group. In scenario 1 and scenario 3 destinations are static and for this, data packets may have high chance to arrive at all destinations. Consequently, the GR for scenario 1 and scenario 3 are higher than the GR for scenario 2.

\subsection{The Performance of FQM under different mobile scenarios and different nodes density}

The mobility of nodes affects the nodes distribution based on the level of nodes density. In this section, the performance of the FQM framework for supporting multimedia applications in MANETs under different nodes density and different mobile scenarios is studied. This section focuses on the effect of the nodes density on the source mobility scenario, destinations mobility scenario and intermediate nodes mobility scenario.

\subsubsection{Packet Delivery Ratio (PDR)}

The PDR as a function node density for the three mobile scenarios is given in Figure 6. The PDR for scenario 3 is slightly decreased when node density increases. This is because most of nodes in the network represent intermediate nodes which are mobile. Increasing node density affects nodes distribution and increases contention and collision between mobile nodes and for this the traffic is congested and the available bandwidth is reduced. For scenario 1, when node density increased, the PDR did not affect because the mobility of source node does not affect node distribution very high. In addition, the slightly increased in PDR for scenario 2 when node density increase can be 
International Journal of Wireless \& Mobile Networks (IJWMN) Vol. 6, No. 1, February 2014

referred to the increase in the number paths from source to destinations. The differences between the PDR for three mobile scenarios with low node density (100 nodes) are discussed in section 4.1.1.

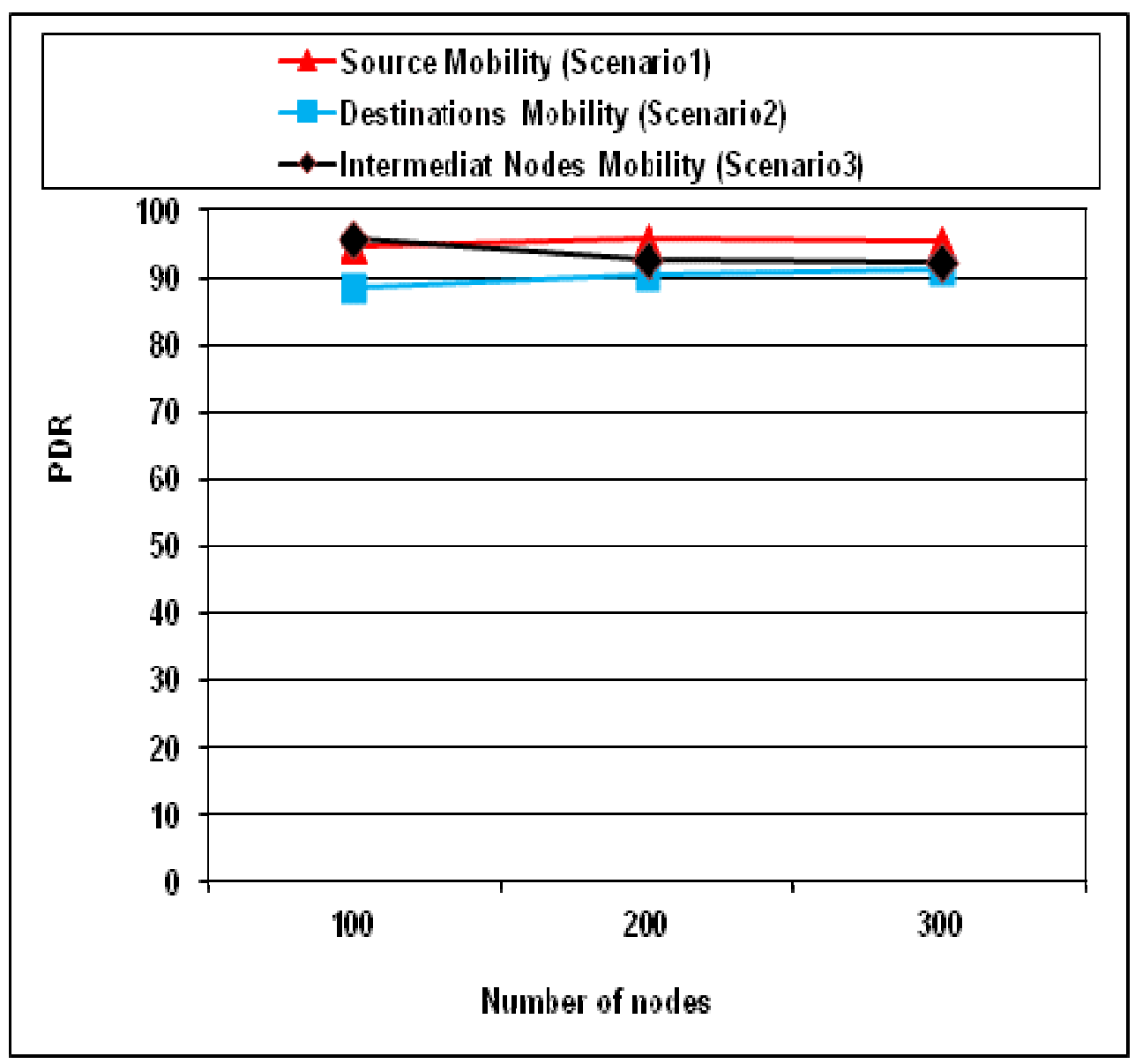

Figure 6. Performance of PDR vs. node density

\subsubsection{Average Latency (AL)}

The AL as a function of node density for the three mobile scenarios is given in Figure 7. The AL for scenario 2 is increased when node density increased. This is because data packets with high latency get alternative paths to arrive at destinations and as a result AL increased and PDR increased as discussed in section 4.2.1. For scenario 1 and scenario 3, the node density did not have high effect on the average latency. The differences between the AL for the three mobile scenarios with low node density (100 nods) are discussed in section 4.1.3. 


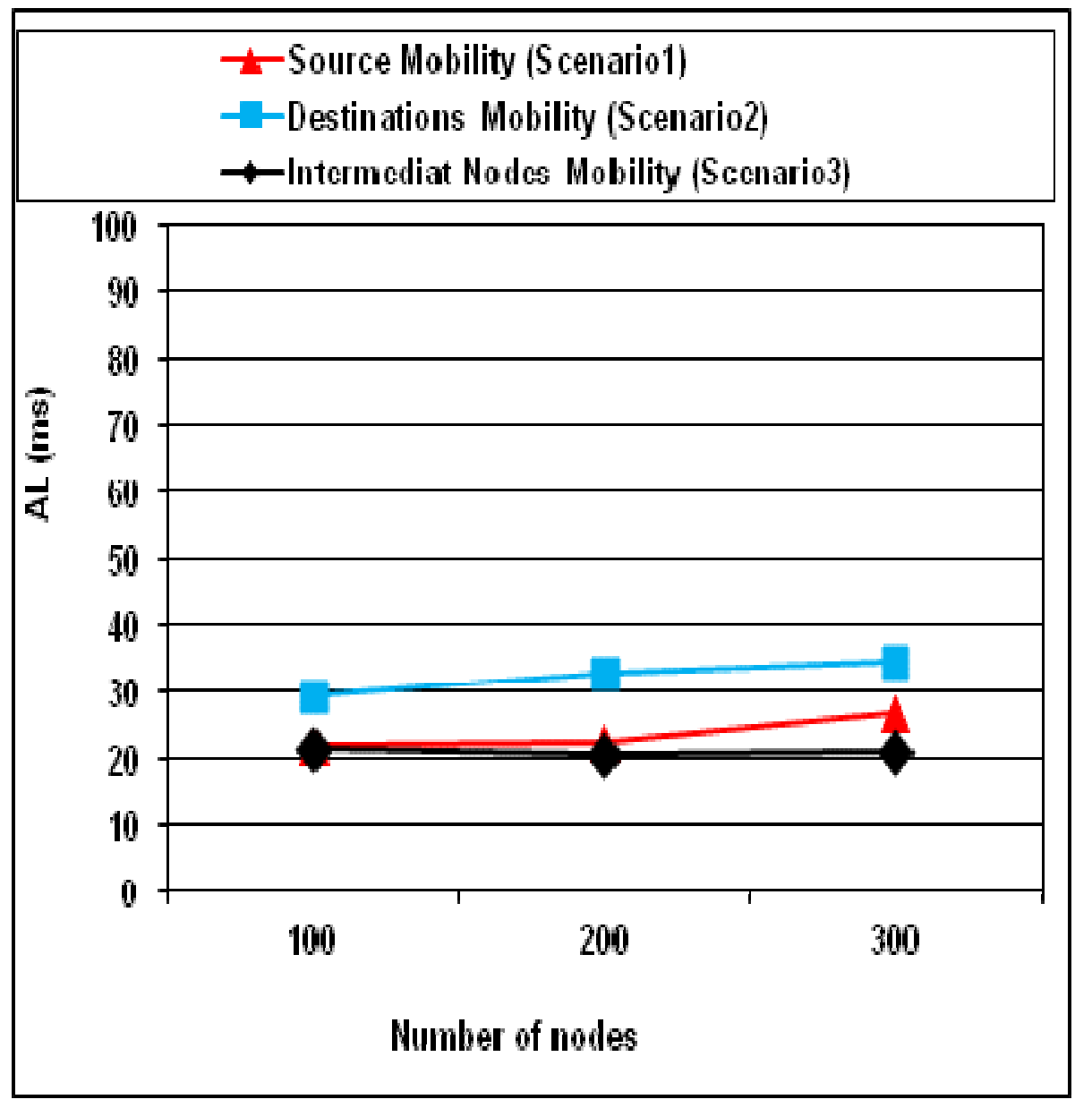

Figure 7. Performance of AL vs. node density

\subsubsection{Jitter}

Figure 8 reflects the jitter as a function of node density for the three mobile scenarios. The figure reflects that jitter for scenario 1 , scenario 2 and scenario 3 are slightly increased when node density increased. This is because number of alternative paths increased and data packets may arrive at destinations through different alternative paths with different latency time. The differences between the jitter for the three mobile scenarios with low node density (100 nodes) are discussed in section 4.1.4. 
International Journal of Wireless \& Mobile Networks (IJWMN) Vol. 6, No. 1, February 2014

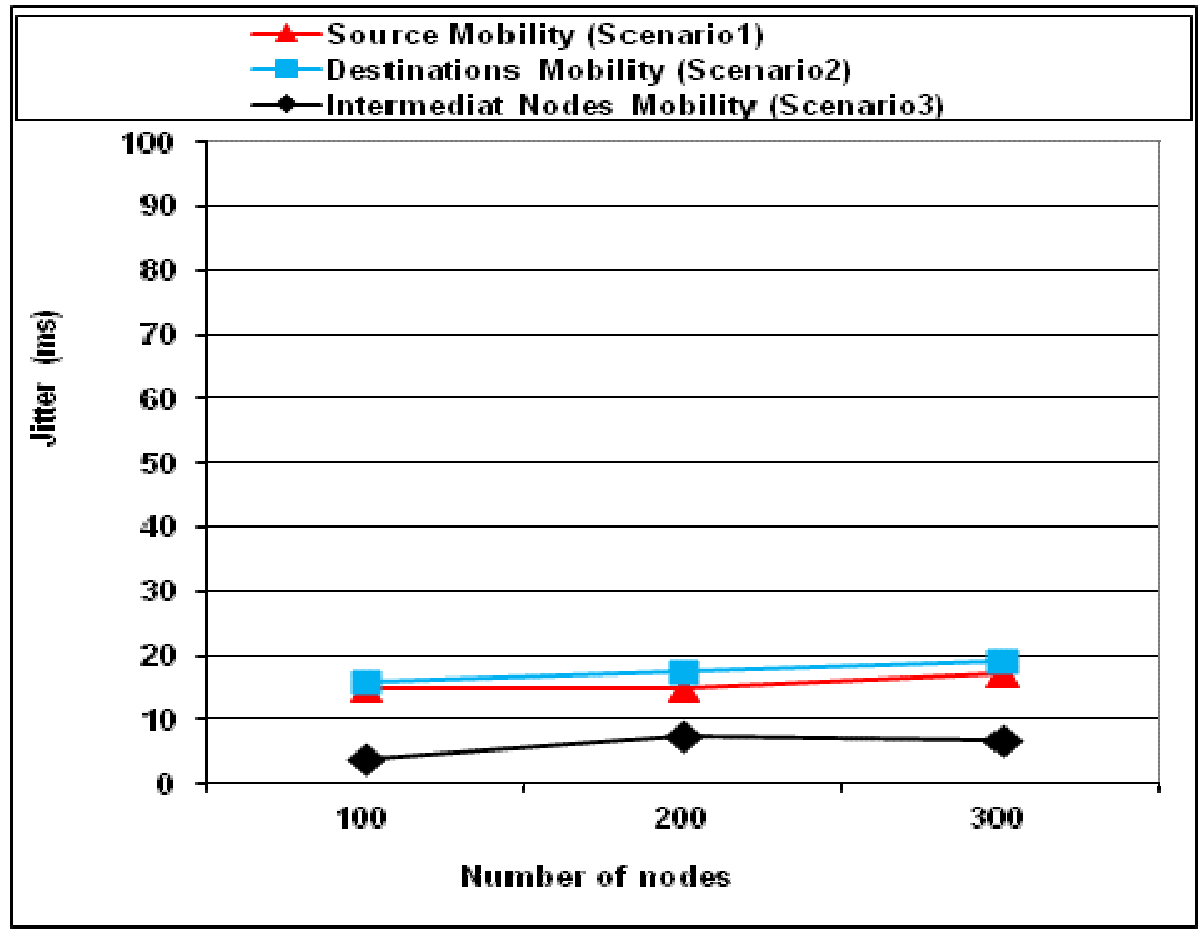

Figure 8. Performance of Jitter vs. node density

\subsubsection{Group Reliability (GR)}

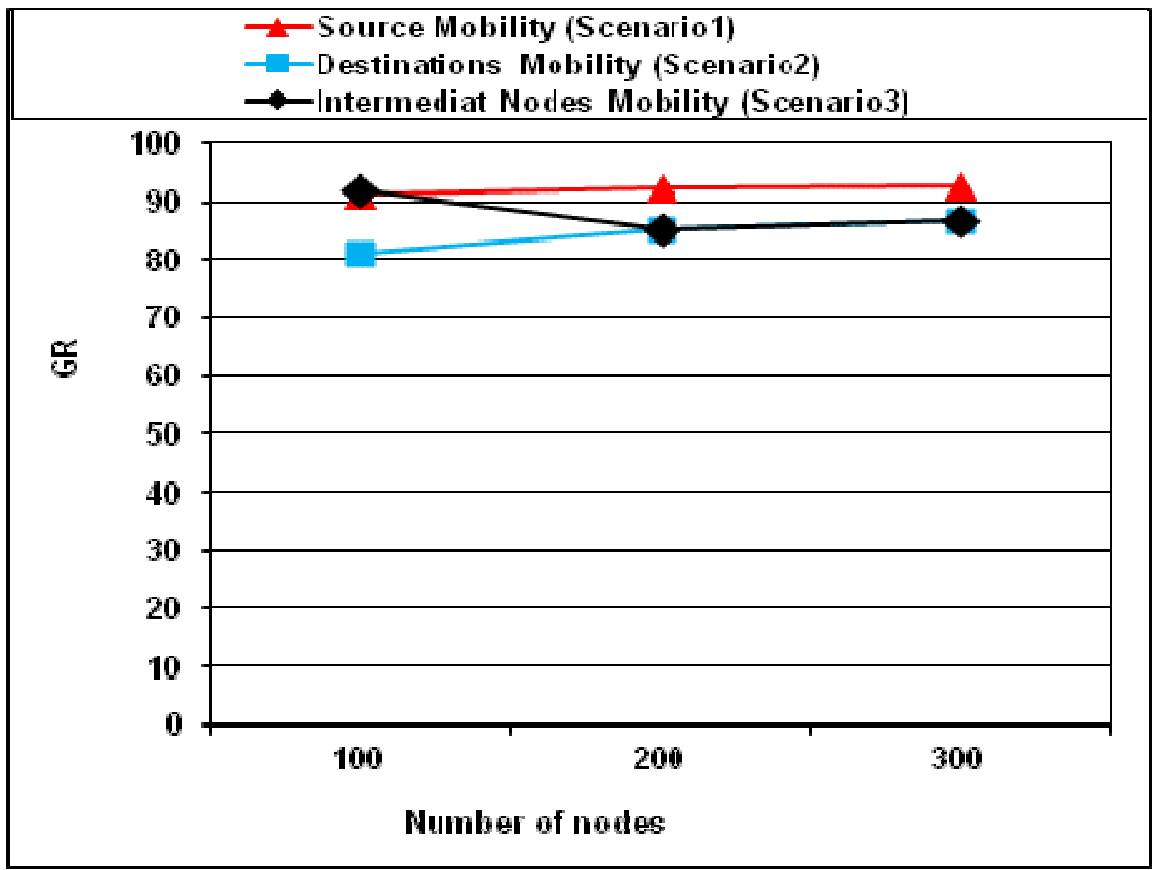

Fig.9: Performance of GR vs. node density 
International Journal of Wireless \& Mobile Networks (IJWMN) Vol. 6, No. 1, February 2014

The GR as a function of node density for the three mobile scenarios is given in Figure 9. The figure shows that GR for scenario 3 decreased when node density increased. This is because the mobility of intermediate nodes with high node density increases contention and collision as discussed in section 4.2.1 and as a result some data packets will be dropped before they arrive at all destinations in the multicast group. The GR for scenario 2 increased when node density increased because mobility of destinations with high node density increases the number of alternative paths and for this data packets may have high chance to arrive at all destinations. The differences between the GR for the three mobile scenarios with low node density (100 nodes) are discussed in section 4.1.5.

\section{CONClusion ANd Future Work}

The mobility of nodes is challenging issue for designers of mobile ad hoc network. Many types of mobile scenarios can be implemented in mobile ad hoc networks and different sets of mobile nodes in MANET may use different mobile scenarios. In this study, the performance of the FQM framework under the source mobile scenario, destination nodes mobile scenario and intermediate nodes mobile scenario is studied. The analysis of simulation results demonstrates that mobility of group of destinations scenario affects the performance of FQM framework much more than mobility of source scenario for multicast routing. Furthermore, the FQM framework can support the applications that based on source mobile scenario better than applications that based on destinations mobile scenario when node density is not high.

In addition, the mobility of intermediate nodes scenario does not have a high effect on the performance of the FQM framework either in PDR, AL, GR or jitter when node density is not high. For general, the mobility of intermediate nodes for QoS multicast protocols that use Forward Nodes does not have a high effect on the performance of the QoS multicast protocols. Furthermore, the QoS multicast protocols that use Forward Nodes to forward data packets are suitable for multimedia applications that based on intermediate nodes mobile scenario. Our future work will focus on implanting the FQM approach with different mobility scenarios under different mobility models to study its performance and efficiency.

\section{REFERENCES}

[1] N. Lakki, A. Ouacha, A. Habbani, M. AJANA EL KHADDAR, M. El Koutbi and J. El Abbadi , (2012) “A New Approach for Mobility Enhancement of OLSR Protocol”, International Journal of Wireless \& Mobile Networks (IJWMN) Vol. 4, No. 1, pp. 117-128.

[2] X. Hong, M. Gerla, G. Pei, and C. Chiang, (1999) "A Group Mobility Model for Ad hoc Wireless Networks", in Proceedings of the 2nd ACM International Workshop on Modeling, Analysis and Simulation of Wireless and Mobile Systems, USA.

[3] T. Ei, S. Diouba, W. Furong and I.Khider, (2008) "Autonomic Group Mobility Model for Mobile Ad hoc Networks", Proceedings of the World Congress on Engineering, London.

[4] B. Zhou, K. Xu and M. Gerla, (2004) "Group and Swarm Mobility Models for Ad Hoc Network Scenarios using Virtual Tracks", IEEE Military Communications Conference, CA.

[5] R. Manoharan and E. Ilavarasan, (2010) "Impact of Mobility On The Performance of Multicast Routing Protocols In MANET", International Journal Of Wireless and Mobile Networks (IJWMN), Vol. 2, No. 2, pp. 110-119.

[6] W. Wei and Avideh Zakhor, (2007) "Multiple Tree Video Multicast over Wireless Ad Hoc Networks", IEEE Transactions on Circuits and Systems for Video Technology, Vol. 17, No. 1, pp. 217.

[7] A. Jardosh, E. M. Belding-Royer, K. C. Almeroth, S. Suri, (2003) "Towards Realistic Mobility Models for Mobile Ad hoc Networks", MobiCom'03, USA.

[8] K. Srikanth and S. Ali Jafar, (2006) "Impact of Mobility on Cooperative Communication", Proceedings of Wireless Communications and Networking Conference, pp. 908-913, las vegas, NV. 
International Journal of Wireless \& Mobile Networks (IJWMN) Vol. 6, No. 1, February 2014

[9] V. Lenders, J. Wagner and M. May, (2006) "Analyzing the Impact of Mobility in Ad Hoc Networks", Proceeding of International Work shop on Multi hop Ad hoc Networks, REALMAN'06, Italy.

[10] A. Alshanyour and U. Baroudi, (2010) "A Simulation Study: the Impact of Random and Realistic Mobility Models on the Performance of Bypass-AODV in Ad Hoc Wireless Networks", EURASIP Journal on Wireless Communications and Networking, Vol. 2010.

[11] P. Joshi, A. Gautam, A. chaudhry, P. Punia, N. Bani, (2012) "Impact of Various Mobility Model and Judgment for Selecting Mode of Network in Different Mobility Situation for Mobile Ad- Hoc Network (MANET)", 1st International Conference on Emerging Technology Trends in Electronics, Communication and Networking, Gujarat.

[12] N. Bhalaji, A. R. Sivaramkrishnan, S. Banerjee, V. sundar and A. Shanmugam, (2009) "Trust Enhanced Dynamic Source Routing Protocol for Ad hoc Networks", International Journal of World Academy of Science, Engineering and Technology Vol. 49, pp. 1074-1079.

[13] C. E. Perkins and P. Bhagwat, (1994) "Highly Dynamic Destination-Sequenced Distance-Vector routing (DSDV) for mobile computers”, In SIGCOMM, pp. 234-244, ACM Press.

[14] M. Zuhairi, H. Zafar and D. Harle, (2012) "The Impact of Mobility Models on the Performance of Mobile Ad Hoc Network Routing Protocol, IETE TECHNICAL REVIEW, Vol. 29, No. 5, pp. 414421.

[15] S.-J. Lee, W. Su and M. Gerla, (2000) “On-Demand Multicast Routing Protocol (ODMRP) for Ad Hoc Networks," Internet Draft, draft-ietf-manet-odmrp-02.txt.

[16] Mario Gerla, Guangyu Pei and Sung-Ju Lee, (1998) "On-Demand Multicast Routing Protocol (ODMRP) for Ad-Hoc Networks", draft-gerla-manet-odmrp-00.txt.

[17] E. M. Royer and C. E. Perkins, (1999) "Multicast Operation of the Ad-hoc On-Demand Distance Vector Routing Protocol," in the Proceedings of the 5th Annual ACM/IEEE International Conference on Mobile Computing and Networking, pp. 207-218.

[18] E. M. Royer and C. E. Perkins, (2000) "Multicast Ad hoc On-Demand Distance Vector (MAODV) Routing," Internet Draft: draft- ietf-manet-maodv-00.txt.

[19] J. G. Jetcheva and D. B. Johnson, (2001) "Adaptive Demand-driven Multicast Routing in Multi-hop Wireless Ad hoc Networks," In Proceedings of the ACM International Symposium on Mobile ad hoc networking and computing, pp. 33-44, USA.

[20] J. Tengviel and K. Diawuo, (2013) "Comparing the Impact of Mobile Nodes Arrival Patterns in Manets using Poisson and Pareto Models", International Journal of Wireless \& Mobile Networks (IJWMN) Vol. 5, No. 5, pp. 179-187.

[21] M. Hasana and L. Hoda, (2004) “Multicast Routing in Mobile Ad Hoc Networks", Kluwer Academic Publishers.

[22] M. Saghir, T. C. Wan, and R. Budiarto, (2006) “A New Cross-Layer Framework for QoS Multicast Applications in Mobile Ad hoc Networks," International Journal of Computer Science and Network Security, (IJCSNS), Vol. 6, No.10, pp. 142-151.

[23] http://pcl.cs.ucla.edu/projects/glomosim.

[24] T. CAMP, J. BOLENG and V. DAVIES, (2002) "A Survey of Mobility Models for Ad hoc Network Research", Wireless Communications \& Mobile Computing, Vol. 2,pp. 483-502.

Author

Mohammed Saghir

Received his B.S from Technology University, Iraq in 1998, M.Sc. from Al Al-Bayt University, Jordan in 2004; and his Ph.D from Universiti Sains Malaysia in 2008 in Computer Sciences. Currently he is working as a senior lecturer in Hodeidah University, Yemen. His current research interests include Mobile Ad hoc networks, QoS multicast routing in MANETs and WiMax.

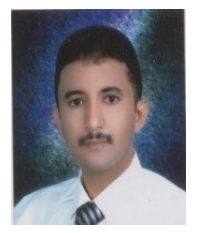

